\title{
Building Information Modelling Execution Plan (BEP): A Comparison of Global Practice
}

\author{
Ahmad Ridzuan Abu Bakar ${ }^{1,2}$, Ahmad Tarmizi Haron ${ }^{1}$ and Rahimi A. Rahman ${ }^{\text {* }}$ \\ ${ }^{I}$ Faculty of Civil Engineering Technology, Universiti Malaysia Pahang, 26300 Gambang, Pahang, Malaysia \\ ${ }^{2}$ Malaysian Public Works Department, Jalan Sultan Salahuddin, 50582 Kuala Lumpur, Malaysia \\ *Email: arahimirahman@ump.edu.my
}

\begin{abstract}
Building Information Modelling (BIM) is a process supported by technologies involving the development and management of 3D digital information model representations of functional and physical of the design. To implement BIM in a project, BIM Implementation Plan or BIM Execution Plan (BEP) must be developed as a planning and monitoring strategy document. Several BEPs have been developed around the world since 2010. However, a BEP should be tailored to the type of project, stakeholder requirement, and project objectives because a comprehensive BEP is a key factor for BIM implementation success. Also, a proper BEP helps stakeholders understand and achieve the underlying objectives in using BIM. In other words, understanding the strategic framework of BEPs is important. Hence, this study identifies and compares the key elements in existing BEPs. To accomplish that aim, twenty BEPs were identified and analysed through the directed content analysis technique. This study provides a benchmarked reference for industry players on existing BEPs. Through a clear description, all parties can understand the requirements in a BEP. Based on the findings and discussions on the twenty (20) samples, BEP is a strategic planning and monitoring document used to ensure that all project deliverables are completed according to the project goals. The document framework shall include the work process, type of information, standard requirements, data exchange process, and roles and responsibilities to develop the project team members' understanding. The framework can guide the project team or stakeholders to identify the key elements and outline contents included in BEP documents. Also, researchers can use the findings for further development and improvement of existing BEPs. Understanding BEPs will ensure better BIM implementation in practice.
\end{abstract}

Indexed Terms- Building information modelling, BIM Execution Plan, BIM Execution Plan Framework.

\section{INTRODUCTION}

Building Information Modelling (BIM) is modelling technology and associated set of processes to produce, communicate and analyse digital information models for the construction life cycle. According to United States National Building Information Model Standard Project Committee [1], BIM is a digital representation of a facility's physical and functional characteristics. Also, BIM is a shared knowledge resource for information about a facility forming a reliable basis for decisions during the facility's earliest conception to demolition (i.e., lifecycle). Currently, the adoption of BIM is increasing worldwide. Governments, authorities, and private sectors are mostly aware of BIM benefits. Therefore, BIM strategic frameworks, policies, and road maps have been developed and regulated by several governments to ensure the success of BIM implementation.

To ensure BIM implementation runs effectively, several reference documents have been developed, such as guidelines, standards, modelling techniques, and work process manuals, including BIM Execution Plans (BEPs). The examples of the documents are: 
- $\quad$ Singapore BIM Guide, 2013 (Singapore)

- NATSPEC National BIM Guide, 2016 (Australia)

- The Guide to Building Information Modelling, 2015 (Belgium)

- AEC (CAN) BIM Protocol, 2014 (Canada)

- BIM Manual Civil Works and Infrastructure Mthojgaard, 2016 (Denmark)

- COBIM Common BIM Requirements, 2012 (Finland)

- National BIM Model Protocol, 2017 (Netherlands)

- CIC Building Information Modelling Standards, 2019 (Hong Kong)

- Design Guidelines BIM Manual Rail Baltica, 2019 (European Union)

- Application Guide BIM Luxembourg CRTI.B, 2018 (Luxembourg)

- The New Zealand BIM Handbook, 2016 (New Zealand)

- $\quad$ Statsbygg BIM Manual 1.2.1, 2013 (Norway)

- $\quad$ AEC (UK) BIM Protocol, 2012 (United Kingdom)

- National BIM Guide for Owners, 2017 (United States)

- Garis Panduan dan Piawaian BIM JKR, 2014 (Malaysia)

BEP is an initial plan document that needs to be developed in the early project stage to ensure better project success [2]. The BEP intends to provide an outline that ensures all parties know the opportunities and responsibilities in implementing BIM for the project [3]. The BEP is a tool to provide a standardised workflow and general guidance for strategic BIM implementation in a holistic approach for a particular project or a group of projects [4]. An organisation or company must choose which BEP standard suits their specific requirement [5]. Also, BEP standards might differ in certain level implementations, such as at organisation, project or team level. Therefore, the main reason for creating a new BEP framework is to ensure the effective integration of BIM in a new type of project [6].

A BEP shall be developed in a holistic approach according to project requirements, including standardised workflow and general guidance for strategic BIM implementation. In other words, construction players must know the best practice on how to develop the BEP according to their organisation and project requirements. According to BIM Project Execution Planning Guide Pennsylvania State University [7], there are four structured steps in developing a BEP:

- Identify high-value BIM goals and uses during project planning, design, construction, and operational phases.

- Design the BIM execution process by creating process maps.

- Define the BIM deliverables in the form of information exchanges.

- Develop the infrastructure in the form of contracts, communication procedures, technology, and quality control to support the implementation.

These steps can guide project teams in developing the project's strategic plan and communication direction. This execution will help the project team understand the project goals, project characteristics, capabilities of project stakeholders, and infrastructure required. For example, Hrdina and Matejka [6] used these steps to develop a BEP for Public-Private Partnership (P3) projects. The newly developed framework can be used by any organisation or company as a reference to develop or modify a BEP for $\mathrm{P} 3$ projects. This shows that developing a BEP requires some guides or references. On the other hand, this process also shows that BEP needs to be developed and modified according to the project implementation method. In other words, understanding the strategic framework of BEPs is important.

This study identifies and compares the key elements in existing BEPs. A framework for BEP development will be presented at the end of the study. For this purpose, twenty (20) BEPs have been identified, compared, and analysed. The findings highlight the elements in BEPs that are necessary for BEP development. Through a clear description, all parties can understand the project objectives, work 
process, roles and responsibilities, information needs, and deliverable requirements. Hence, this BEP framework can be used as a reference for the development and improvement of BEP. It will increase the knowledge of BIM implementation in new projects. The findings of this study also offer a comprehensive list of parameters for researchers and practitioners to develop tools that promote BIM project implementation success. This study contributes to the body of knowledge by identifying a framework and improvement requirements that shall increase the effectiveness of BIM. Hence, this lesson will assist the industry player in improving the success rate of BIM execution.

\section{BIM EXECUTION PLANS}

Organisations have described the definition and purpose of BEP production. The subsequent content illustrates those definitions and purposes from several BEPs.

NATSPEC National BIM Guide [8]. The BIM Management Plan (BMP) is a formal document that defines how the project will be executed, monitored, and controlled concerning BIM. It is required that BMP be developed to provide a master information/data management plan and assignment of roles and responsibilities for model creation and data integration at project initiation.

Garis Panduan BIM JKR, Public Works Department Malaysia [9]. The BEP shall be developed as a planning and monitoring strategy document for BIM implementation. The BEP intends to provide an outline that will ensure all parties know the opportunities and responsibilities of projects that implement BIM.

GSA Building Information Modelling Guide, US General Services Administration (GSA) [10]. The BEP is developed to provide a master information/data management plan and assign roles and responsibilities for model creation and data integration at project initiation. The BEP aligns the project acquisition strategy needs and requirements with GSA technical standards, team member skills, construction industry capability, and technology maturity.

The New Zealand BIM Handbook, MBIE [3]. The BEP intends to provide an outline that will ensure all parties know the opportunities and responsibilities of projects that implement BIM. The plan defines the objectives of using BIM on the project. It sets goals, objectives, and people's responsibilities and outlines how the process will be executed in the project's life cycle.

Based on existing BEPs, it was found that all the documents consist of the common BEP chapters. However, some of the BEP's structure outlines were different depending on its priority. Meanwhile, some of the BEP's comprise additional chapters for better understanding. Hence, further analyses and comparisons of the guidelines will illustrate available patterns that exist in BEP frameworks.

\section{METHODOLOGY}

\subsection{Qualitative Research Technique and Comparative Analysis}

This study embarks on a qualitative approach that is content analysis, sample comparison, and interviews. In this study, the study produces a BEP framework that applies to the construction industry. The approach was qualitative as the inductive approach. Other studies are also using this technique to compare available content in the existing state of the art and practice, including assessment techniques for BIM skills [11], enforcement procedures for environmental laws [12], and optimization techniques for rehabilitating water distribution networks [13]. Twenty (20) BEPs were selected and gathered for analysis to develop the BEP framework. The detailed steps involve:

- Exploration of the elements in each BEP's.

- Execute the comparison analysis and develop it in table form. 
- Summarise the conclusion of all consistent key elements contained in all BEPs.

- Develop the BEP theoretical framework based on the key elements.

- Preparing and validating the report of findings.

\subsection{Source of Material}

Many countries implement BIM in their projects. However, in this study, only twenty (20) BEPs were selected and gathered for analysis. The BEPs are:

- BIM Project Execution Planning Guide, Pennsylvania State University, USA [7]

- $\quad$ NATSPEC National BIM Guide, Australia [8]

- CPIx BIM Execution Plan, UK [14]

- BIM Essential Guide for BIM Execution Plan, Building and Construction Authority, Singapore [15]

- Garis Panduan BIM JKR, Public Works Department, Malaysia [9]

- The Guide to Building Information Modelling Association of Major Belgian Contractors (ADEBVBA), Belgium [16]

- BIM Execution Plan Template, Metrolinx Toronto, Canada [17]

- National BIM Guide, The BIM Academy, South Africa [18]

- MIT Design Standard BIM Execution Plan, Massachusetts Institute of Technology, USA [19]

- The New Zealand BIM Handbook, MBIE, New Zealand [3]

- GSA Building Information Modelling Guide, US General Services Administration, USA [10]

- National Model BIM Implementation Plan, BIR WG BIM Protocol, Netherlands [20]

- Resource Centre for Building Technologies and Innovation (CRTI-B) Luxembourg [21]

- Smithsonian Institution (SI) Facilities BIM Guidelines, USA [22]

- BIM Execution Plan, MARU 360 Group, Ananda Development, Thailand [23]

- BIM Guidelines and Standards, The University of South Florida, USA [24]

- E/A Design Division BIM Standard, The Port Authority of NY \& NJ, USA [25]

- BIM Manual Design Guideline Rail Baltica, RB Rail, European Union [26]

- Queensland Health BIM Execution Plan, State of Queensland, Brisbane, Australia [27]

- BIM Execution Plan, Catenda AS, Norway [28]

\section{RESULTS AND DISCUSSION}

Based on the analysis among twenty (20) BEPs, it was found that almost all BEPs have a similar theoretical framework. Most of the BEPs were prepared based on Pennsylvania State University BEP (2010) framework because it is one of the world's earliest BEPs. Although the framework was similar, some of the content presentation and chapter titles were different. The BEP outlines including project information, project goals/BIM objectives/BIM uses roles and responsibilities, BIM process design, BIM information exchange, collaboration procedures, model structure, quality control, technology infrastructure needs, and project deliverables. The comparison results of the BEP key elements in each BEP as shown in Table 1. 
Table 1: The key elements in each BEP

\begin{tabular}{|c|c|c|c|c|c|c|c|c|c|c|c|c|c|c|c|c|c|c|c|c|}
\hline References & 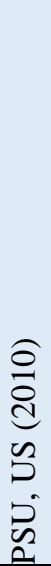 & 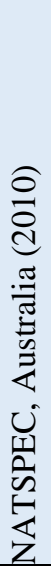 & 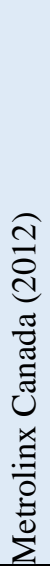 & 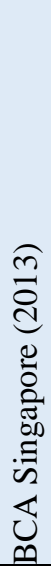 & 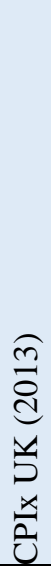 & 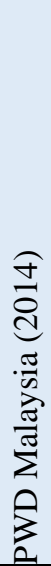 & 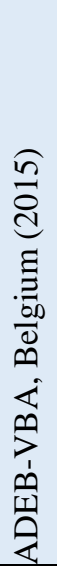 & 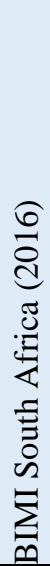 & 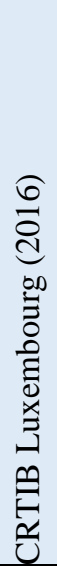 & 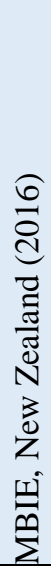 & 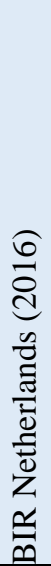 & 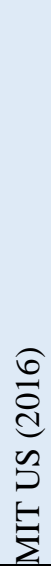 & 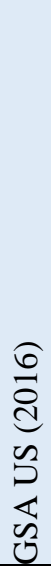 & 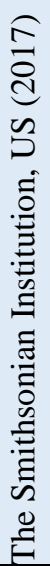 & 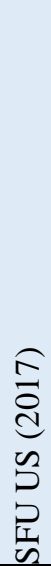 & 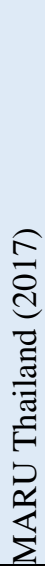 & 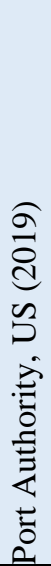 & 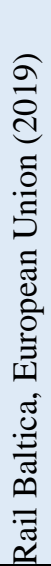 & 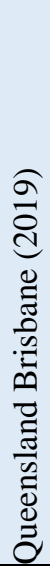 & 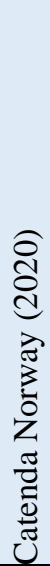 \\
\hline a) Project Information & 1 & 1 & l & 1 & 1 & I & 1 & I & I & I & 1 & I & 1 & 1 & I & I & 1 & 1 & I & I \\
\hline Project Name & 1 & 1 & 1 & 1 & I & 1 & 1 & I & I & 1 & l & I & I & I & I & I & I & I & 1 & I \\
\hline Project Scope Brief & I & I & I & I & I & 1 & $\mathrm{x}$ & I & I & I & l & l & I & I & I & I & I & I & I & $\mathrm{x}$ \\
\hline Type of Contract & 1 & 1 & 1 & 1 & 1 & 1 & $\mathrm{x}$ & $\mathrm{x}$ & 1 & 1 & 1 & 1 & 1 & 1 & 1 & 1 & 1 & $\mathrm{x}$ & $\mathrm{x}$ & $\mathrm{x}$ \\
\hline b) Project Goals/BIM Objectives & I & l & l & I & l & I & I & I & I & I & I & l & I & I & I & I & I & I & 1 & I \\
\hline Goal Description & I & I & 1 & I & I & I & 1 & I & I & I & 1 & I & I & I & I & I & I & I & I & 1 \\
\hline BIM Uses & / & / & / & / & $\mathrm{x}$ & I & $\mathrm{x}$ & $\mathrm{x}$ & / & / & I & I & / & / & / & / & / & I & $\mathrm{x}$ & I \\
\hline c) Roles and Responsibilities & I & I & I & l & I & I & l & I & l & l & 1 & l & I & I & I & l & I & l & 1 & I \\
\hline BIM / Model / Process Manager & I & / & / & I & $\mathrm{x}$ & I & I & I & I & / & I & $\mathrm{x}$ & I & I & I & I & I & I & I & 1 \\
\hline BIM Lead Coordinator & $\mathrm{x}$ & / & $\mathrm{x}$ & $\mathrm{x}$ & $\mathrm{x}$ & I & $\mathrm{x}$ & $\mathrm{x}$ & $\mathrm{x}$ & l & I & $\mathrm{x}$ & $\mathrm{x}$ & $\mathrm{x}$ & l & $\mathrm{x}$ & l & $\mathrm{x}$ & $\mathrm{x}$ & I \\
\hline BIM Coordinator & I & l & I & l & $\mathrm{x}$ & I & l & $\mathrm{x}$ & l & l & I & $\mathrm{x}$ & $\mathrm{x}$ & $\mathrm{x}$ & $\mathrm{x}$ & l & l & I & $\mathrm{x}$ & I \\
\hline BIM Modeller / Author / Tech. & $\mathrm{x}$ & $\mathrm{x}$ & 1 & 1 & 1 & I & 1 & $\mathrm{x}$ & 1 & $\mathrm{x}$ & $\mathrm{x}$ & $\mathrm{x}$ & $\mathrm{x}$ & $\mathrm{x}$ & $\mathrm{x}$ & 1 & 1 & 1 & 1 & I \\
\hline BIM CAD / Support Group & $\mathrm{x}$ & 1 & $\mathrm{x}$ & $\mathrm{x}$ & $\mathrm{x}$ & 1 & I & $\mathrm{x}$ & 1 & $\mathrm{x}$ & $\mathrm{x}$ & $\mathrm{x}$ & $\mathrm{x}$ & $\mathrm{x}$ & $\mathrm{x}$ & 1 & 1 & $\mathrm{x}$ & $\mathrm{x}$ & I \\
\hline Project / Construction Manager & 1 & 1 & 1 & 1 & 1 & 1 & 1 & 1 & $\mathrm{x}$ & 1 & 1 & 1 & 1 & 1 & 1 & 1 & 1 & 1 & 1 & 1 \\
\hline Designers & 1 & 1 & $\mathrm{x}$ & 1 & 1 & 1 & 1 & I & 1 & 1 & 1 & 1 & 1 & 1 & I & 1 & 1 & 1 & 1 & 1 \\
\hline Draught person & I & I & $\mathrm{x}$ & $\mathrm{x}$ & $\mathrm{x}$ & $\mathrm{x}$ & $\mathrm{x}$ & $\mathrm{x}$ & $\mathrm{x}$ & $\mathrm{x}$ & $\mathrm{x}$ & $\mathrm{x}$ & $\mathrm{x}$ & $\mathrm{x}$ & $\mathrm{x}$ & $\mathrm{x}$ & $\mathrm{x}$ & $\mathrm{x}$ & $\mathrm{x}$ & $\mathrm{x}$ \\
\hline Quantity Surveyor / Cost Planner & $\mathrm{x}$ & I & $\mathrm{x}$ & 1 & $\mathrm{x}$ & I & I & $\mathrm{x}$ & $\mathrm{x}$ & I & 1 & $\mathrm{x}$ & $\mathrm{x}$ & $\mathrm{x}$ & $\mathrm{x}$ & 1 & $\mathrm{x}$ & $\mathrm{x}$ & 1 & $\mathrm{x}$ \\
\hline Surveyor & $\mathrm{x}$ & $\mathrm{x}$ & $\mathrm{x}$ & l & $\mathrm{x}$ & $\mathrm{x}$ & $\mathrm{x}$ & $\mathrm{x}$ & $\mathrm{x}$ & $\mathrm{x}$ & 1 & $\mathrm{x}$ & $\mathrm{x}$ & $\mathrm{x}$ & $\mathrm{x}$ & $\mathrm{x}$ & $\mathrm{x}$ & $\mathrm{x}$ & $\mathrm{x}$ & $\mathrm{x}$ \\
\hline Building Users / Owners / Client & I & l & $\mathrm{x}$ & I & $\mathrm{x}$ & $\mathrm{x}$ & 1 & 1 & I & I & 1 & $\mathrm{x}$ & / & $\mathrm{x}$ & 1 & l & I & $\mathrm{x}$ & $\mathrm{x}$ & I \\
\hline
\end{tabular}




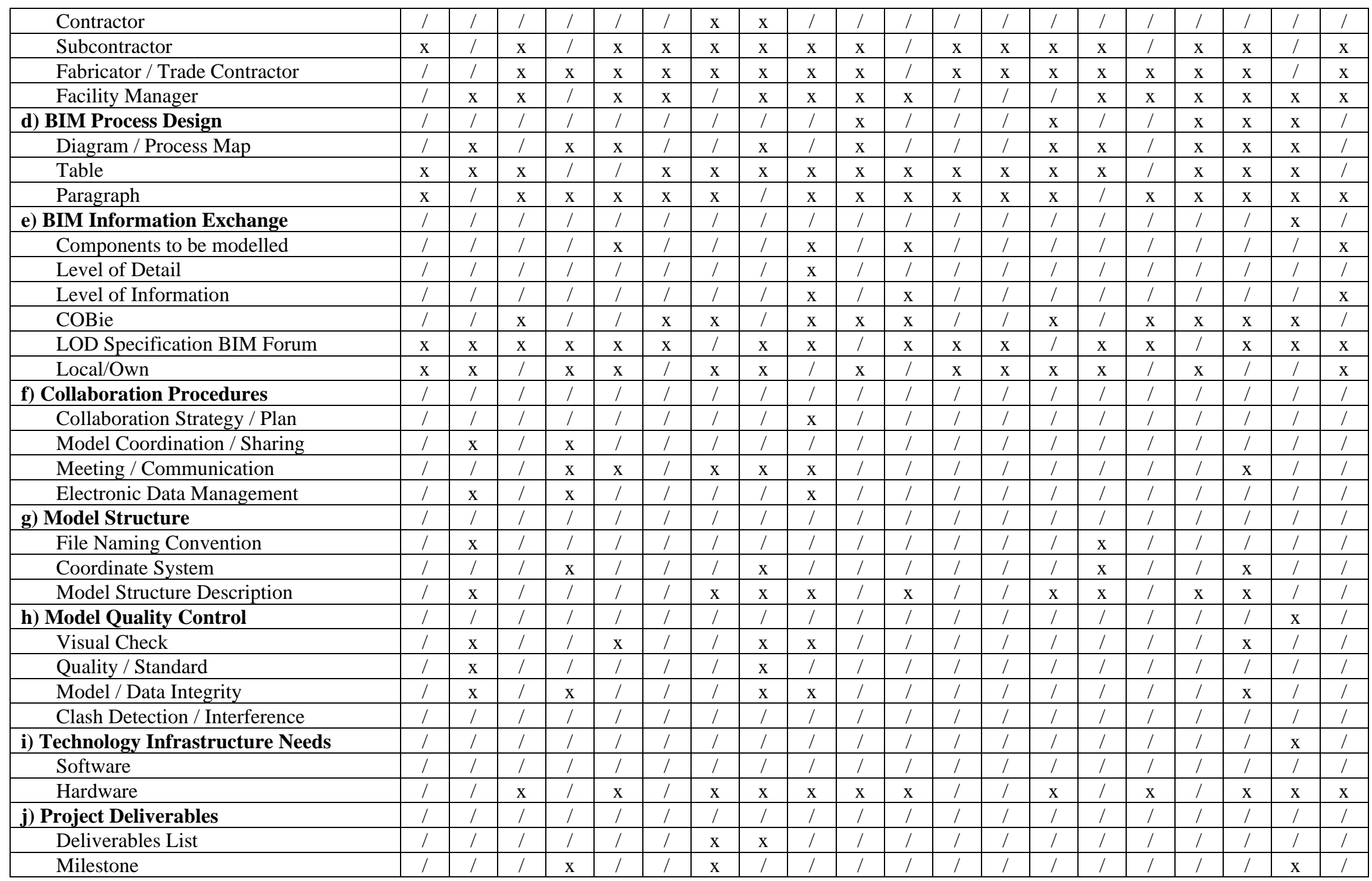




\subsection{Project Goals/BIM Objectives}

This section explains the target and objective of using BIM in the projects. It describes how BIM is used to achieve specific project goals at different project stages [15]. This section should document the strategic value and specific uses for BIM on the project as defined by the project team in the initial planning procedures. The objectives and uses for BIM shall be defined at the start of the project. It will be difficult to implement additional functionality in the BIM models tater at the post-contract stage [29]. The selection of BIM uses shall be based on the objectives and goal descriptions. However, the determination of BIM uses depends on human resource capabilities and technology infrastructures, such as hardware and software. Most countries or organisations designated BIM use as a requirement to be specified in the BEP template. Normally, the potential BIM uses a table showing the relation between goal description, BIM objectives, project stages, and responsible parties. Although specified in their BIM Guidelines or BIM Standards, four (4) countries or organisations did not specify BIM uses in the BEP template, including the BEP Template of CPIx UK, ADEB-VBA Belgium, BIMI South Africa, and Queensland Brisbane.

\subsection{Roles and Responsibilities}

The effective BEP includes a schedule and contact information for all parties. This provides accountability and direction for contributors, encouraging everyone to continue to be responsible for their tasks [30]. One of the primary tasks is to define the project team members for the execution process throughout the various stages of the project. The BIM Team should define the working relationships between roles so that lines of authority are clear and facilitate efficient resolution of issues as they arise [8]. Although many people were involved in the project, not all team members have been listed in the contact list or roles and responsibilities tables. Some organisations have mentioned the BIM CAD / Support Group or BIM Modelling Expert as a team member. This group is responsible for guiding the team members on software tool applications or BIM work processes, especially for beginners. Meanwhile, the Facility Manager is only involved if the organisation plan to roll out the BIM implementation in the facility management stage. The involvement shall be started in the early stage to define the information need for facility management.

\subsection{BIM Process Design}

This section should illustrate the detail of the execution process. Process maps diagram will help the team members understand a detailed plan for implementing each BIM uses, including specific information exchanges for each activity for the entire execution plan. The plan should include the overview map of the BIM Uses, a detailed map of each BIM Use, and a description of elements on each map [7]. The effective presentation of BIM processes will explain the whole BIM process in the project implementation. The type of presentation, including a diagram, map, workflow, table, or text, depends on organisation arrangement. For example, Pennsylvania State University US BEP Template has provided the BIM overview map and detailed process maps for BIM that showed the relation between processes, BIM uses information exchange, and responsible parties. Building and Construction Authority Singapore has provided the BIM Use Case Template in table form, which showed the connection between BIM use cases, authors, and users. The essential task, quality task, and deliverables expected also have been described in BIM use cases. Meanwhile, South Florida University, US has provided a detailed description in Section 7: Model Objectives and Application, including explaining the objectives, model roles, and responsibilities. Based on the finding, only five (5) organisations or countries did not describe the BIM detail process explanation, including the BEP Template of MBIE New Zealand, The Smithsonian Institution US, Port Authority US, Queensland Brisbane, and Rail Baltica European Union.

The BIM work process showed the workflow diagram or table of model development through BIM Process Mapping from the beginning until the end of deliverables production, including: 
- The parties who involved in supplying and receiving the information.

- The input needed by the model's author.

- The parties whose involvement in modelling, collaboration, and coordination process.

- The modelling, collaboration, and coordination processes.

- The workflow of information exchange and the type of file format.

- The output that the model's author shall deliver.

According to the BIM Forum [31], the Level of Development (LOD) is the degree to which the element's geometry and attached data have been thought through the degree to which project team members may rely on the information when using the model. LOD consists of two categories, which are Level of Detail and Level of Information. Most of the BEP Template has provided the list of building components that shall be modelled. The details of the geometry and shape of the component, which need to be created, become more detailed according to the LOD of the project. Several BEP Template refers to the Level of Development Specification Guide (LOD Forum) to explain LOD requirements for each building component in every stage. The detail of information needed depended on the client's project brief, which shall be defined in the initial stage of the project. Based on findings, most of the Levels of Information have been detailed in BEP Template or other supportive reference documents. For example, Pennsylvania State University, US BEP Template only mentioned construction, engineering, and record information as a general required information in Attachment 4 - Information Exchange Worksheet. For further description, Pennsylvania State Computer Integrated Construction has produced a Planning Guide for Facility Owners to explain the details of the Level of Information [32].

Meanwhile, NATSPEC Australia has provided BIM Object Element Matrix, which shown the requirement of detailed information in all components through multiple software formats, including Revit and ArchiCAD. The information required is referred to as Construction Operation Building Information Exchange (COBie). According to National Building Specification UK, COBie is a nonproprietary data format for the publication of a subset of BIM focused on delivering asset data distinct from geometric information. To standardise the type of information and arrangement, nine (9) organisations or countries were referred to COBie, and four (4) organisations or countries were referred to Uniformat OmniClass in Level of Development Specification [31].

\subsection{Collaboration Procedures}

The model elements and detail required to implement each BIM use should be clearly defined in the information exchange requirements because some of the team members need different information in certain project stages. This section will describe how the project team will collaborate, including Items such as the Common Data Environment (CDE) for managing project information, communication methods, transfer, record storage, and others (New Zealand BIM Handbook, November 2016). This procedure is key to project information management, consisting of data sharing, gathering, transfer, conversion, and storage. This collaboration also depends on the technical capabilities and cost provided.

\subsection{Model Structure}

According to Charles Pankow Foundation and BIM Forum [30], the BEP should document the relationship between object, building, campus, and state plane coordinate systems. This section will describe and produce a simple diagram to show how the model is developed and separated either by zone, floor, building element, system, or discipline. A properly structured model is important because space and building area calculations are derived from the model [3]. The BIM Manager shall also define the standard naming conventions for models following the approved format or standard by the client. Files must be named in this exact format to avoid system conflicts when drawings are transferred to the Smithsonian Institution's archival system [22]. 


\subsection{Model Quality Control}

This section will explain the procedure for ensuring that the project participants meet the defined requirements that should be developed and monitored throughout the project. The effectiveness of BIM implementation depends on the quality of the deliverables. In this context, the BIM model shall be developed according to the BIM guidelines, standards, and requirements. The integrity of the information will assist all team members in fully utilised the BIM model in every stage of the project. The geometry and non-geometry data shall be accurate and reliable. The quality and integrity of the models shall accord to the modelling technique in their standard. Modelling mistakes will impact the quantity of the materials, the accuracy of information designs conflict, and drawing interpretation. Four significant types of checking were listed in most BEP templates, such as visual, quality, integrity, and interference checking.

\subsection{Project Deliverables}

The list of BIM deliverables for the project and the format in which the information will be delivered shall be recorded. A milestone is a significant event that project team members shall achieve. All deliverables need to be submitted to the client according to the projection and milestones in the work programme schedule. Team members shall conduct risk management or mitigation action if the submission of the deliverables cannot meet the target date. An example of the deliverable events is model submission, clash analysis reports, and coordination meetings.

\section{CONCLUSION}

Based on the findings and discussions on the twenty (20) BEPs, it was found that the BEP framework was a key factor for the project's BEP development. The document framework shall include the strategy, process, information, infrastructure, personal and standards to develop the project team members' understanding (Figure 1).

Several organisations or countries separate the BEPs in the design and construction stage or pre-contract and post-contract, such as BEP Queensland Health BEP and CPIx UK. According to BEP Queensland Health, the Design BEP or DBEP will always be created before developing the Construction BEP or CBEP. The DBEP and federated model will be used by tendering contractors to inform the development of the CBEP. To get a comprehensive comparison, several BEPs from organisations or countries have been selected. The BEPs were tailored to individual project or organisation requirements. Most BEPs are detailed and comprehensive, but some are relatively simple

Meanwhile, the outline and elements in the BEPs depend on the BEP developer. The work process and information exchange also differ between BEPs, including workflow diagrams, tables, and matrix schedules. Overall, the BEP document will define and facilitate the exchange of information between all participants and establish coordination procedures and consistency among all trades. It will also organise all BIM-related activities around a collaborative design philosophy and emphasise BIM's role in the beneficial reuse of project data throughout the whole lifecycle. This will ensure that all information provided by all participants is reliably and precisely developed in each stage of design, construction, and facility operation and maintenance throughout the project lifecycle.

These findings highlight the necessary elements in a BEP for future developments. Several improvement requirements were also discussed in previous sections and should be added to increase the effectiveness of the BEP framework. Thus, this study's lesson would help the industry to improve the success rate of BIM execution. 


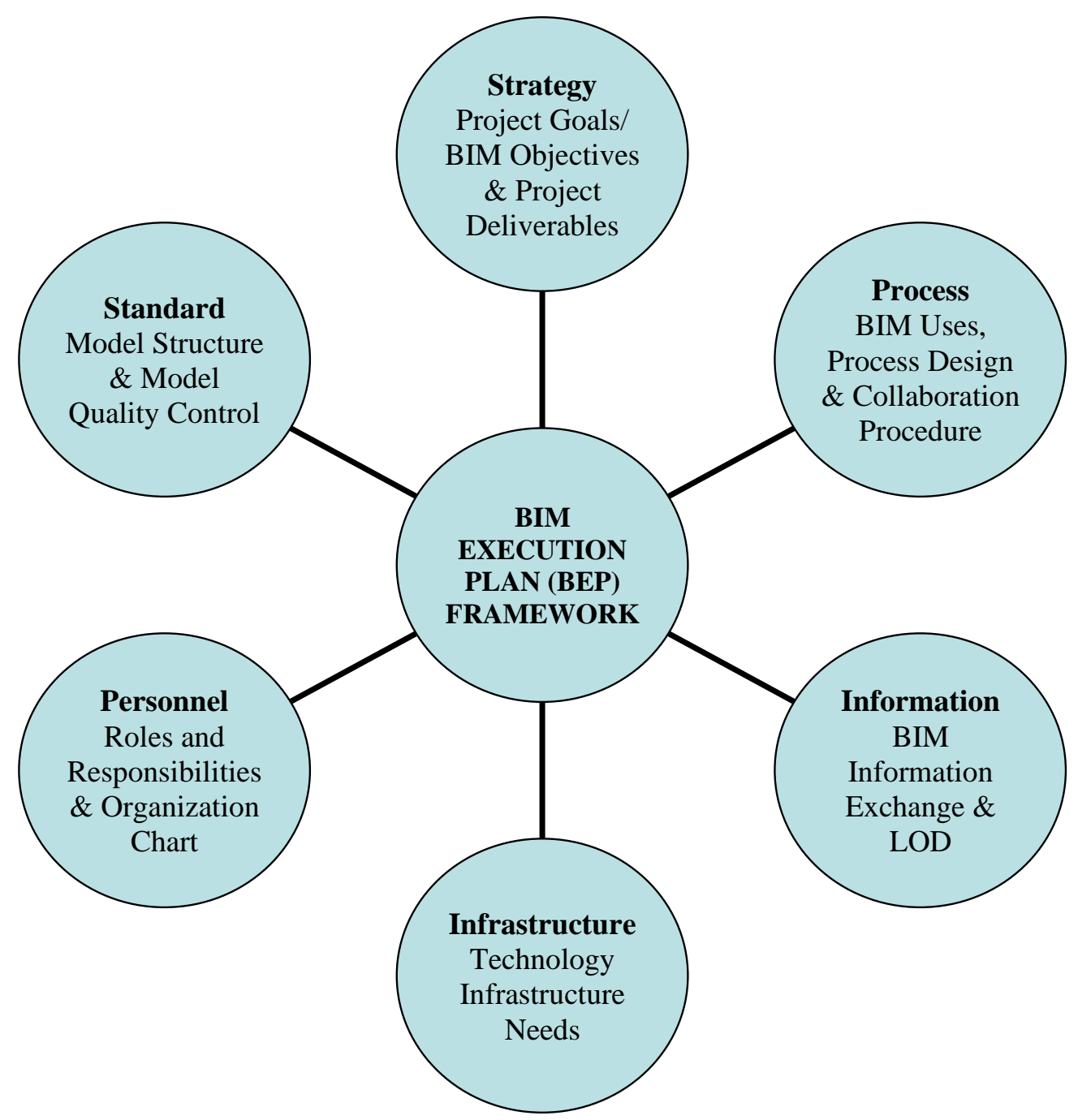

Figure 1: BIM Execution Plan (BEP) Framework

\section{ACKNOWLEDGMENT}

This work is supported by Universiti Malaysia Pahang [RDU190382]. The authors would like to thank the editors and anonymous reviewers whose invaluable comments and suggestions substantially helped improve this paper's quality. The authors are also thankful to the industry practitioners that participated in this work.

\section{REFERENCES}

[1] NIBS. United States national building information modeling standard: Version 2. National Institute of Building Sciences. 2012.

[2] Ibrahim, F. S., Shariff, N. S., Esa, M., Rahman, R.A. "The barriers factors and driving forces for bim implementation in Malaysian AEC companies." J. Adv. Res. Dynam. Control Syst 11, $275-$ 284, 2019.

[3] BIM Acceleration Committee. New Zealand BIM Handbook BIM. BIM Acceleration Committee, Productivity Partnership and BRANZ Building Research Levy, 2015. 
[4] McArthur, J.J., Sun, X. "Best practices for BIM execution plan development for a public-private partnership design-build-finance-operate-maintain project." Built Environ, 149, pp.119-130, 2015.

[5] Ahmad, A.M., Demian, P., Price, A. "Building information modelling implementation plans a comparative analysis." In Proceedings of 28th Annual ARCOM Conference 33-42, 2012.

[6] Hrdina, O., Matejka, P. "BIM execution plan in Czech Republic." Bus. IT, 6, pp.17-23, 2016.

[7] Pennsylvania State Department of Architectural Engineering. BIM Project Execution Planning Guide and Template - Version 2.12010.

[8] NATSPEC. NATSPEC BIM Management Plan Template (v1.0), NATSPEC, 2012.

[9] PWD. Garis Panduan BIM JKR Malaysia, Public Works Department (PWD), Malaysia, 2015

[10] GSA. US General Services Administration, 2019.

[11] Rahman, R. A., Ayer. S. K. "Assessment strategies for building information modeling skills in problem-based learning pedagogics." In Proceedings of 6th CSCE/CRC International Construction Specialty Conference. 2017.

[12] Ng C. C., Rahman R. A., Ahmad S. W. "Factors affecting the enforcement of environmental regulation: the case of erosion and sediment control plan." In IOP Conference Series: Earth and Environmental Science, vol. 682, no. 1, p. 012046. IOP Publishing, 2021.

[13] Farouk A. M., Romali N. S., Rahman R. A., Seman M. A. "Optimization Techniques for Rehabilitating Water Distribution Networks." In IOP Conference Series: Earth and Environmental Science, vol. 641, no. 1, p. 012019. IOP Publishing, 2021.

[14] Construction Project Information (CPIx) Committee (UK), Post Contract-Award Building Information Modelling (BIM) Execution Plan (BEP), 2013.

[15] Singapore Building and Construction Authority (SBCA). Singapore BIM Essential Guide for BIM Execution Plan (Version 2), 2013.

[16] ADEB. The Guide to Building Information Modelling Association of Major Belgian Contractors (ADEB-VBA), 2015.

[17] Metrolinx. BIM Execution Plan Template, Metrolinx Toronto Canada, 2016.

[18] The BIM Academy. National BIM Guide, The BIM Academy, South Africa, 2016.

[19] MIT. MIT Design Standards BIM Execution Plan v6.0, Department of Facilities Massachusetts Institute of Technology (MIT), 2016.

[20] BIR Working Group. National Model BIM Implementation Plan, BIR Working Group BIM Protocol, Netherlands, 2016.

[21] CRTI-B. Application Guide BIM Luxembourg, Resource Centre for Building Technologies and Innovation (CRTI-B), 2016.

[22] Smithsonian Facilities. Smithsonian Facilities BIM Guidelines, Smithsonian Facilities, 2017.

[23] Ananda Development. MARU 360 BIM Execution Plan, Ananda Development, Thailand, 2017.

[24] University of South Florida. BIM Guidelines and Standards, University of South Florida, 2018.

[25] The Port Authority. E/A Design Division BIM Standard, The Port Authority, New York \& New Jersey, 2019.

[26] Rail Baltica. BIM Manual Design Guideline Rail Baltica, RB Rail, European Union, 2019.

[27] State of Queensland. Queensland Health BIM Execution Plan, State of Queensland, Brisbane Australia, 2019.

[28] Catenda. BIM Execution Plan, Catenda AS, Norway, 2020.

[29] CIC. CIC BIM Standards General, Construction Industry Council, Hong Kong, August 2019.

[30] Charles Pankow Foundation - BIM Forum BIM. Project Execution Plan (BxP) Guide, An Introduction for Those New to BIM Version 1.00, 2019.

[31] BIM Forum. Level of Development (LOD) Specification for Building Information Models and Data. BIM Forum, 2020.

[32] Pennsylvania State Department of Architectural Engineering. The Uses of BIM - Classifying and Selecting BIM Uses Version 0.9, Penn State Computer Integrated Construction, 2013. 